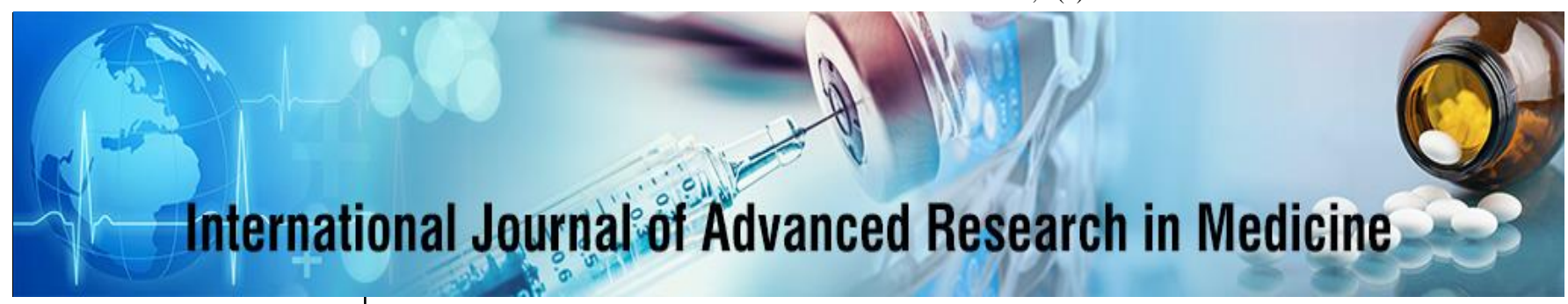

E-ISSN: 2706-9575

P-ISSN: 2706-9567

IJARM $2020 ; 2(1): 67-70$

Received: 11-11-2019

Accepted: 19-12-2019

Dr. Suresh Patel

MD Medicine, Metro Hospital and Cancer Research Center Jabalpur, Madhya Pradesh, India

\section{A clinical investigation to assess the association between serum bilirubin levels and coronary artery disease: A case-control study}

\section{Dr. Suresh Patel}

DOI: https://doi.org/10.22271/27069567.2020.v2.i1a.124

\begin{abstract}
Aims: The aim of the present study was to assess the association between serum bilirubin levels and coronary artery disease in comparison with controls without coronary artery disease.

Material and methods: A cross-sectional study was conducted in the Department of medicine, India from Jan 2019 to Jan 2020. Total of 160 subjects were included in the study with 80 cases and 80 controls. General and systemic examination was conducted on all study subjects including laboratory investigations like complete blood count, renal function test, lipid profile, viral markers such as HBsAG, HCVIgM and liver function test which includes total bilirubin, direct and indirect, liver enzymes, albumin and globulin levels.

Results: The mean age among the cases male and female respectively was $62.76 \pm 8.2$ and $63.85 \pm 8.3$ and controls group were $61.85 \pm 8.4$ and $62.38 \pm 8.5$ years male and female respectively. $60 \%$ were male and $40 \%$ female in case group and $63.75 \%$ patients were male and $36.25 \%$ patients were female in control. The most common risk factors for CVD like diabetes, hypertension, smoking, obesity and family history of CVD was found to be slightly higher among the cases than the control groups but it was not found to be statistically significant and it proves that the controls were matched for almost all the risk factors for CVD except for dyslipidemia which was found to be significantly higher among the CVD patients than the controls. The mean duration of CVD was $4.4 \pm 2.8$ years. The various liver function test parameters were compared between the cases and controls it was found that the serum bilirubin levels which includes total bilirubin, direct bilirubin and indirect bilirubin was found to be lower among the case group compared to the control group and this difference was found to be statistically significant, whereas the other parameters like SGOT, SGPT and GGT levels did not show much difference between the case and control groups and the difference in values were not statistically significant.

Conclusion: The relationship between the decreased serum bilirubin levels and the event of CAD; in this manner, bilirubin level can fill in as a prognostic factor, together with other significant factors for recognizing an individual who is in the peril of coronary artery disease.
\end{abstract}

Keywords: CAD, bilirubin level, liver enzymes

\section{Introduction}

Coronary artery disease (CAD) is one of the most common causes of death worldwide. In 2010, about 7 out of total 53 million deaths were due to ischemic heart disease ${ }^{[1]}$. Heart muscle as an oxidative tissue and with constant activity is one of the tissues susceptible to oxidative damage caused by reactive oxygen species such as superoxide, hydrogen peroxide and hydroxyl ${ }^{[2]}$. Oxidative stress plays an important role in the pathogenesis of degenerative diseases or chronic such as atherosclerosis, diabetes and cancer ${ }^{[3,4]}$. Oxidative stress as a result of an imbalance between the production of free radicals and reactive oxygen species on the one hand and the antioxidant defense system on the other hand. In other words, aerobic biological systems in order to cope with free radicals and reactive oxygen species, antioxidant defense mechanisms designed to neutralize the deleterious effects of these factors attacker, or to a minimum ${ }^{[4]}$. New scientific studies have suggested that bilirubin is a potential physiological antioxidant that can be an important protective role against atherosclerosis, CAD and inflammation take over ${ }^{[5]}$. Many factors increase the risk of CAD, including low serum bilirubin level, which is associated with increased risk of CAD [6]. Several studies have shown that bilirubin, the final product of hem metabolismhas potent antioxidant capacity ${ }^{[7]}$.
Corresponding Author: Dr. Suresh Patel MD Medicine, Metro Hospital and Cancer Research Center Jabalpur, Madhya Pradesh, India 
A study performed on healthy individuals. Dividing them according to serum bilirubin levels into 3 groups of low, intermediate and high, has shown that high bilirubin level prevents coronary flow reserve impairment, microvascular dysfunction and probably coronary atherosclerosis ${ }^{[8]}$. Epidemiologic studies have indicated that the total bilirubin level is inversely related to diabetes mellitus, hypotension, $\mathrm{CAD}$ and metabolic syndrome ${ }^{[9,10]}$. Atherosclerosis and inflammation are associated with free oxygen and peroxyl radicals' formation ${ }^{[11,12]}$. Arterial protective responses and adjustment against oxidative stress have important roles in atherosclerosis prevention ${ }^{[13]}$. Studies have shown that different forms of circulating bilirubin and its precursor, biliverdin, have the ability to remove the reactive forms of oxygen. They also prevent the oxidation of low-densitylipoprotein particles and monocyte chemotaxis all which are considered as stages of atherosclerosis [14, 15]. Very few studies in India had been conducted to prove the association between serum bilirubin levels and coronary artery disease and so the present study was undertaken to assess the association between these two variables by comparing it with a control group. The aim of the present study was to assess the association between serum bilirubin levels and coronary artery disease in comparison with controls without coronary artery disease.

\section{Material and Methods}

A cross-sectional study was conducted in the Department of medicine, India from Jan 2019 to Jan 2020, after taking the approval of the protocol review committee and institutional ethics committee. After taking informed consent detailed history was taken from the patient or the relatives. The technique, risks, benefits, results and associated complications of the procedure were discussed with all patients.

\section{Inclusion criteria}

- Patients with evidence of coronary artery disease by ECG, ECHO

\section{Exclusion criteria}

- Patients with symptoms of congestive cardiac failure

- Chronic kidney disease,

- Chronic liver disease, autoimmune diseases,

- COPD and malignancy

\section{Methodology}

Controls were selected matched with age, gender and other co-morbid conditions. Total of 160 subjects were included in the study with 80 cases and 80 controls. General and systemic examination was conducted on all study subjects including laboratory investigations like complete blood count, renal function test, lipid profile, viral markers such as HBsAG, HCVIgM and liver function test which includes total bilirubin, direct and indirect, liver enzymes, albumin and globulin levels. A 12 lead ECG and a transthoracic echocardiogram was performed for all patients. Total serum bilirubin was measured in the laboratory by spectrophotometry method. In the Jendrassik-Gr of allied methods, total bilirubin is reacted with diazotized sulfanilic acid in an acidic medium to form azobilirubin. The absorbance of the azo pigment is then measured as direct bilirubin and the total bilirubin is measured after treatment with alkaline tartrated solution, which shifts the maximum absorption of the azo pigment towards longer wavelength.

Statistical analysis: All the data were entered and analysed using SPSS version 22. Mean and standard deviation was derived for all the parametric variables and the parametric variables between the two groups were compared using unpaired student $t$ - test and comparison between the frequencies was done by using chi-square test considering $p<0.05$ as statistically significant

\section{Results}

The entire study subjects were divided into two groups of 80 cases (with CVD) and 80 controls. Table 1 shows the mean age and sex distribution of the study subjects. Majority of the patients were in the age group between 50-60 years. The minimum age was 39 and the maximum age was 69years. The mean age among the cases male and female respectively was $62.76 \pm 8.2$ and $63.85 \pm 8.3$ and controls group were $61.85 \pm 8.4$ and $62.38 \pm 8.5$ years male and female respectively. $60 \%$ were male and $40 \%$ female in case group and $63.75 \%$ patients were male and $36.25 \%$ patients were female in control group and So, it shows that the cases and controls did not show any significant difference with respect to age and gender which implies that the controls were age and sex matched.

Table 1: Age and sex wise distribution of the study subjects

\begin{tabular}{|c|c|c|c|c|c|}
\hline Age group & \multicolumn{2}{|c|}{ Cases $=\mathbf{8 0}$} & \multicolumn{2}{c|}{ Controls=80 } & P value \\
\hline & Males $=$ 48 & Females $=32$ & Males $=\mathbf{5 1}$ & Females $=\mathbf{2 9}$ & \multirow{2}{*}{$>0.05$} \\
\hline Mean \pm SD & $63.8 \pm 7.9$ & $64.6 \pm 8.1$ & $62.3 \pm 8.4$ & $63.7 \pm 8.6$ & \\
\hline
\end{tabular}

The most common risk factors for CVD like diabetes, hypertension, smoking, obesity and family history of CVD was found to be slightly higher among the cases than the control groups but it was not found to be statistically significant and it proves that the controls were matched for almost all the risk factors for CVD except for dyslipidemia which was found to be significantly higher among the CVD patients than the controls (Table 2).

Table 2: Prevailing risk factors for CVD among study subjects

\begin{tabular}{|c|c|c|c|}
\hline Risk factors & Cases $(\mathbf{n}=\mathbf{8 0})$ & Controls $(\mathbf{n}=\mathbf{8 0})$ & P value \\
\hline Diabetes & $26(32.5 \%)$ & $22(27.5 \%)$ & 0.327 \\
\hline Hypertension & $43(53.75 \%)$ & $33(41.25 \%)$ & 0.148 \\
\hline Smoking & $29(36.25 \%)$ & $27(33.75 \%)$ & 0.855 \\
\hline Family history of CVD & $33(41.25 \%)$ & $25(31.25 \%)$ & 0.266 \\
\hline Obesity & $21(26.25 \%)$ & $15(18.75 \%)$ & 0.177 \\
\hline Dyslipidemia & $50(62.25 \%)$ & $33(41.25 \%)$ & 0.002 \\
\hline
\end{tabular}

Table 3: Distribution of the cases based on their duration of CVD.

\begin{tabular}{|c|c|c|c|}
\hline Duration of CVD & Frequency & Percentage & \multirow{2}{*}{ Mean \pm SD } \\
\hline Below 3 years & 16 & $20 \%$ & \\
\cline { 1 - 3 } 3 - 5 years & 40 & $50 \%$ & \multirow{2}{*}{$4.4 \pm 2.8$} \\
\hline 5 - 7 years & 17 & $21.25 \%$ & \\
\hline Above 7 years & 7 & $8.75 \%$ & \\
\cline { 1 - 3 } Total & 80 & $100 \%$ & \\
\hline
\end{tabular}

The duration of CVD among the cases varied from 1 years to 10 years with majority of the subjects' duration was between 3 and 5 years and the mean duration was $4.4 \pm 2.8$ years. The patients' CVD status was confirmed by history, ECG findings and ECHO reports (Table 3). 
The various liver function test parameters were compared between the cases and controls it was found that the serum bilirubin levels which includes total bilirubin, direct bilirubin and indirect bilirubin was found to be lower among the case group compared to the control group and this difference was found to be statistically significant, whereas the other parameters like SGOT, SGPT and GGT levels did not show much difference between the case and control groups and the difference in values were not statistically significant (Table 4).

Table 4: Comparison of the liver function test parameters between the CVD patients and the controls.

\begin{tabular}{|c|c|c|c|}
\hline LFT & $\begin{array}{c}\text { Cases } \\
(\mathbf{M e a n} \pm \mathbf{S D})\end{array}$ & $\begin{array}{c}\text { Controls } \\
(\mathbf{M e a n} \pm \mathbf{S D})\end{array}$ & P value \\
\hline Total bilirubin & $0.92 \pm 0.06$ & $1.19 \pm 0.24$ & $<0.001$ \\
\hline Direct bilirubin & $0.25 \pm 0.06$ & $0.51 \pm 0.11$ & $<0.001$ \\
\hline Indirect bilirubin & $0.67 \pm 0.11$ & $0.85 \pm 0.15$ & $<0.001$ \\
\hline SGOT (IU/L) & 16 & 29 & 0.588 \\
\hline SGPT (IU/L) & 28 & 34 & 0.205 \\
\hline GGT (IU/L) & 33 & 30 & 0.306 \\
\hline
\end{tabular}

For all the CVD patients an echocardiogram was performed and their ejection fraction was recorded and it was correlated with the serum bilirubin levels, authors found a perfect linear correlation between the ejection fraction and serum bilirubin levels, as the ejection fraction decreases the serum bilirubin levels was also decreasing and all the serum bilirubin parameters were found to be very low in patients with ejection fraction $<50 \%$ when compared to patients with ejection fraction $>60 \%$ and this association was found to be statistically significant $(p<0.05)$ (Table 5).

Table 5: Association and correlation between serum bilirubin levels and the ejection fraction among the CVD patients.

\begin{tabular}{|c|c|c|c|c|c|}
\hline Serum bilirubin & $\begin{array}{c}>60 \%(\mathbf{n}= \\
\mathbf{2 0})\end{array}$ & $\begin{array}{c}\mathbf{5 0 - 6 0 \%} \\
(\mathbf{n = 4 3})\end{array}$ & $\begin{array}{c}<\mathbf{5 0 \%} \\
(\mathbf{n = 1 7})\end{array}$ & $\begin{array}{c}\mathbf{P} \\
\text { value }\end{array}$ & $\begin{array}{c}\mathbf{r} \\
\text { value }\end{array}$ \\
\hline $\begin{array}{c}\text { Total bilirubin } \\
(\text { Mean } \pm \text { SD) }\end{array}$ & $1.3 \pm 0.29$ & $0.87 \pm 0.18$ & $0.75 \pm 0.22$ & $<0.001$ & 0.921 \\
\hline $\begin{array}{c}\text { Direct bilirubin } \\
\text { (Mean } \pm \text { SD) }\end{array}$ & $0.44 \pm 0.13$ & $0.34 \pm 0.14$ & $0.23 \pm 0.05$ & $<0.001$ & 0.879 \\
\hline $\begin{array}{c}\text { Indirect bilirubin } \\
\text { (Mean } \pm \text { SD })\end{array}$ & $0.74 \pm 0.27$ & $0.67 \pm 0.15$ & $0.62 \pm 0.09$ & $<0.001$ & 0.926 \\
\hline
\end{tabular}

\section{Discussion}

Atherosclerosis is considered to be the most common underlying cause for the coronary artery disease (CAD), which is the major cause of mortality worldwide both in developed and developing countries. Whereas on the other hand antioxidants are the predominant adaptive responses by the arterial vasculature in response to the oxidative stress thereby preventing the atherosclerosis ${ }^{[16]}$. Bilirubin, being a toxic waste product formed during heme catabolism is in fact a potent physiological antioxidant that provides important protection against atherosclerosis and inflammation ${ }^{[17]}$. A particular enzyme namely the heme oxygenase (HO) is a stress inducible enzyme in the heme catabolism which plays an important role in cell defense mechanism against oxidative injury.

The products of the catabolic reaction, i.e. bilirubin, carbon monoxide and iron have a protective role. The other important role of bilirubin, the natural antioxidants are the inhibition of vascular cell adhesion molecule VCAM-1 preventing the proliferation of the smooth muscle cells and the trans endothelial migration of the leucocytes ${ }^{[18]}$.

Plasma bilirubin inversely correlated with risk factors of CAD- smoking, diabetes and obesity, thus emphasizing the oxidative stress underlying in them, but in present study authors did not observed such correlation as authors matched most of the risk factors between the cases and controls. Inverse relationship between the presence of CAD and circulatory total bilirubin was first observed by Schwertner et al. ${ }^{[19]}$.

Male gender is one of the most important risk factors for CAD. In this study the mean age among the cases male and female respectively was $62.76 \pm 8.2$ and $63.85 \pm 8.3$ and controls group were $61.85 \pm 8.4$ and $62.38 \pm 8.5$ years male and female respectively. $60 \%$ were male and $40 \%$ female in case group and $63.75 \%$ patients were male and $36.25 \%$ patients were female in control group and So, it shows that the cases and controls did not show any significant difference with respect to age and gender which implies that the controls were age and sex matched. we also matched other comorbidities thereby removing the confounding factors responsible for the lowering of bilirubin as a result of the oxidative stress and other mechanisms ${ }^{[20]}$.

Present study found a significant inverse association between serum bilirubin and CAD in comparison with control, bilirubin levels found to be significantly lower in $\mathrm{CAD}$ patients in comparison with the controls $(p<0.001)$ and a similar type of results was also quoted by Taban SM et $a l$, and in their study they had also found a significant association between the bilirubin levels and the severity of CAD by doing an angiogram. ${ }^{21}$ So it seems that higher bilirubin level has a protective effect against coronary artery stenosis (CAS).

The present study among 80 CAD patients and 80 healthy controls confirmed the results of several previous epidemiological studies that low serum bilirubin levels were associated with increased risk for coronary events [22, 23, 24]. A recent study in patients with peripheral arterial disease (PAD) revealed similar results showing a clear association between low bilirubin concentrations and PAD ${ }^{[25]}$. Present study showed a higher level of mean total bilirubin in males in comparison to females, but the difference was not statistically significant, however lower levels of bilirubin in females may be attributed to the influence of estrogens. This may relate to the increased secretion of bilirubin through the induction of UDP- glucuronil transferasa enzyme in liver. Estrogens also decrease LDL level, increase HDL level and reduce LDL oxidation ${ }^{[26]}$. Recently, low serum bilirubin levels have been proposed as a useful biomarker to predict cardiovascular risk and suggests that bilirubin acts as a potent physiologic antioxidant and anti-inflammatory agent. Studies have shown that elevated serum bilirubin concentrations provide important protection against atherosclerotic diseases ${ }^{[16]}$. Several authors have suggested that bilirubin plays a potential role in inhibition of lipid oxidation ${ }^{[27]}$. An inverse correlation between the presence of coronary artery disease, peripheral arterial disease, carotid intima-media thickness and bilirubin has been reported in several studies. Subnormal levels of plasma bilirubin are associated with premature coronary artery disease and cardiovascular morbidity ${ }^{[28]}$. In a previous study, the 3-year incidence of coronary artery disease was significantly lower in patients with Gilbert syndrome ${ }^{[29]}$. 
This study showed a significant relation between ejection fraction with total serum bilirubin the ejection fraction showed a descending trend as serum bilirubin level decreased and a similar type of results was also quoted by Taban SM et al. ${ }^{[21]}$.

\section{Conclusion}

This study has demonstrated a critical relationship between the decreased serum bilirubin levels and the event of CAD; in this manner, bilirubin level can fill in as a prognostic factor, together with other significant factors for recognizing an individual who is in the peril of coronary artery disease.

\section{Reference}

1. Lozano R, Naghavi M, Foreman K, Lim S, Shibuya K, Aboyans $\mathrm{V}$, et al. Global and regional mortality from 235 causes of death for 20 age groups in 1990 and 2010: A systematic analysis for the Global Burden of Disease study 2010. Lancet 2012;380(9859):2095-128.

2. Rahbar S, Ahmadiasl N. Effect of Long Term Regular Resistance Exercise on Heart Function and Oxidative Stress in Rats. Ardebil University of Medical Sciences Journal 2013;12(3):256-64.

3. Karajibani M, Montazerifar F, Hashemi M, Bolouri A, Dikshit M. Study of oxidative stress in patients with angina pectoris admitted to cardia care unit (CCU) at khatam-al-Anbia hospital in zahedan. Zahedan Journal of Research in Medical Sciences 2013;15(3):20-5.

4. Steghamati A, Zarban A, Doosti M. Evaluation of antioxidant status and oxidative stress index in patients with Type II diabetes mellitus. Iran $\mathbf{J}$ Endocrinol Metabolism 2002;3(4):239-45.

5. Nabatchian F, Einollahi N, Boroomand MA, Abbasi S. Can bilirubin be an indicator for predicting coronary artery disease? Payavarde Salamat 2014;7(2):91-100.

6. Lingenhel A, Kollerits B, Schwaiger JP, Hunt SC, Gress $\mathrm{R}$, Hopkins PN, et al. Serum bilirubin levels, UGT1A1 polymorphisms and risk for coronary artery disease. Exper Gerontol J 2008;43(12):1102-107.

7. Stocker R, Glazer AN, Ames BN. Antioxidant activity of albumin-bound bilirubin. Proceedings of the National Academy of sciences of the United States of America 1987;84:5918-922.

8. Gullu H, Erdogan D, Tok D, Topcu S, Caliskan M, Ulus $\mathrm{T}$, et al. High serum bilirubin concentrations preserve coronary flow reserve and coronary microvascular functions. Arterioscler Thromb Vasc Biol 2005;25(11):2289-294

9. Hopkins PN, Wu LL, Hunt SC, James BC, Vincent GM, Williams RR. Higher serum bilirubin is associated with decreased risk for early familial coronary artery disease. Arterioscler Thromb Vasc Biol 1996;16(2):250-55.

10. Giral P, Ratziu V, Couvert P, Carrie A, Kontush A, Girerd $\mathrm{X}$, et al. Plasma bilirubin and gammaglutamyltransferase activity are inversely related in dyslipidemic patients with metabolic syndrome: relevance to oxidative stress. Atherosclerosis 2010;210(2):607-13.

11. Hansson GK, Robertson AK, Soderberg-Naucler C. Inflammation and atherosclerosis. Annu Rev Pathol Mech Dis 2006;1:297-329.

12. Berliner JA, Navab M, Fogelman AM. Atherosclerosis: basic mechanisms. Oxidation, inflammation, and genetics. Circulation 1995;91(9):2488-496.
13. Ghem C, Sarmento-Leite RE, de Quadros AS, Rossetto $S$, Gottschall C. Serum bilirubin concentration in patients with an established coronary artery disease. Int Heart J 2010;51(2):86-91.

14. Neuzil J, Stocker R. Free and albumin-bound bilirubin are efficient co-antioxidants for alphatocopherol, inhibiting plasma and low density lipoprotein lipid peroxidation. J Biol Chem 1994;269(24):16712-6719.

15. Rigato I, Ostrow JD, Tiribelli C. Bilirubin and the risk of common non-hepatic diseases. Trends Mol Med 2005;11(6):277-83.

16. Mayer M. Association of serum bilirubin concentration with risk of coronary artery disease. Clin Chem 2000;46(11):1723-7.

17. Morita T. Heme oxygenase and atherosclerosis. Arterioscler Thromb Vasc Biol 2005;25(9):1786- 95.

18. Neuzil J, Stocker R. Free and albumin-bound bilirubin are efficient co-antioxidants for alpha- tocopherol, inhibiting plasma and low density lipoprotein lipid peroxidation. J Bio Chem 1994;269(24):16712-9.

19. Schwertner HA, Jackson WG, Tolan G. Association of low serum concentration of bilirubin with increased risk of coronary artery disease. Clin Chem 1994;40(1):18-23

20. Ghem C, Sarmento-Leite RE, de Quadros AS, Rossetto $S$, Gottschall CA. Serum bilirubin concentration in patients with an established coronary artery disease. Int Heart J 2010;51(2):86- 91.

21. Taban SM, Golmohammadi A, Parvizi R, Kezerlou AN, Separham A, Hosnavi Z. The relation of serum bilirubin level with coronary artery disease based on angiographic findings. Crescent J Med Biol Sci 2015;2(4):130-4.

22. Troughton JA, Woodside JV, Young IS, Arveiler D, Amouyel $\mathrm{P}$, Ferrières $\mathrm{J}$, et al. Bilirubin and coronary heart disease risk in the Prospective Epidemiological Study of Myocardial Infarction (PRIME). Eur J Cardiovasc Prevent Rehabilitation 2007;14(1):79-84.

23. Novotny L, Vitek L. Inverse relationship between serum bilirubin and atherosclerosis in men: a meta- analysis of published studies. Exp Biol Med (Maywood) 2003;228(5):568-71.

24. Djoussé L, Rothman KJ, Cupples LA, Levy D, Ellison RC. Effect of serum albumin and bilirubin on the risk of myocardial infarction (the Framingham offspring study). Am J Cardiol 2003;91(4A):485-8.

25. Rantner B, Kollerits B, Anderwald-Stadler M, KleinWeigel $\mathrm{P}$, Gruber I, Gehringer A, et al. Association between the UGT1A1 TA-repeat polymorphism and bilirubin concentration in patients with intermittent claudication: results from the CAVASIC study. Clin Chem. 2008;54(5):851-7.

26. Freeman R. Hormone replacement therapy (estrogen and progesterone): is it necessary for heart disease prevention?. Preventive Cardiol 2000;3(1):21- 4.

27. Stocker R, Glazer AN, Ames BN. Antioxidant activity of albumin-bound bilirubin. Proceed National Academy Sci 1987;84(16):5918-22.

28. Ishizaka N, Ishizaka Y, Takahashi E, Yamakado M, Hashimoto H. High serum bilirubin level is inversely associated with the presence of carotid plaque. Stroke 2001;32(2):580-3

29. Vítek L, Jirsa Jr M, Brodanová M, Kaláb M, Mareček Z, Danzig V, et al. Gilbert syndrome and ischemic heart disease: a protective effect of elevated bilirubin levels. Atherosclerosis 2002;160(2):449-56. 